\title{
Anti-relaxation therapy in GORD
}

\section{J Tack, D Sifrim}

\section{Reducing transient LOS relaxations in patients with gastro-oesophageal reflux disease}

G astro-oesophageal reflux disease (GORD), defined as the presence of symptoms or lesions that can be attributed to the reflux of gastric contents into the oesophagus, is one of the most common disorders affecting the gastrointestinal tract. Gastric acid is essential in the development of GORD and the duration of oesophageal acid exposure is a major determinant of the severity of reflux oesophagitis. ${ }^{1}$ This is the reason why proton pump inhibitors, with their strong acid suppressing action, have brought relief to the majority of these patients, although most GORD patients do not have increased gastric acid secretion.

The pathophysiology of GORD is multifactorial, including alterations in the volume, composition, or distribution of gastric contents, anatomical and/or motor dysfunction of the antireflux barrier at the gastro-oesophageal junction, impaired clearance mechanisms, and defective resistance to injury at the mucosal level. Acid suppressive therapy addresses one of the pathophysiological mechanisms-that is, gastric acid volume-without affecting others such as the non-acid component of the gastric contents or the function of the antireflux barrier. In spite of the efficacy of acid suppressive therapy, there have been many attempts to target other aspects of GORD pathophysiology, such as basal lower oesophageal sphincter (LOS) pressure, oesophageal body motility, or gastric emptying. Unfortunately, most of these approaches have been clinically disappointing.

The prerequisite for the development of acid reflux events is the occurrence of functional and/or anatomical failure of the antireflux barrier at the gastrooesophageal junction. In healthy subjects and in patients with mild to moderate GORD, reflux occurs mainly during transient LOS relaxations (TLOSRs). ${ }^{3-7}$ TLOSRs are LOS relaxations not induced by swallowing, which occur mainly during the first two postprandial hours. In patients with more severe GORD and in those with a hiatal hernia, although TLOSRs account for the majority of reflux episodes, a greater proportion occurs during absent basal LOS pressure and swallow induced LOS relaxations.
Transient LOS relaxations are a neural reflex, triggered mainly by distention of the proximal stomach and organised in the brain stem, with efferent and afferent pathways travelling in the vagus nerve, activating an intramural inhibitory neurone which releases nitric oxide to relax the LOS. ${ }^{9}$ Pharmacological intervention to reduce TLOSRs has been proposed as a non-surgical strategy to improve the antireflux barrier in patients with GORD, and several agents that inhibit the occurrence of TLOSRs were identified, including atropine, cholecystokinin $_{\mathrm{A}}$ receptor antagonists, morphine, and nitric oxide synthase inhibitors. ${ }^{10}$ However, an unfavourable pharmacological profile precluded clinical application of these agents. More recently, the gamma aminobutyric acid (GABA) receptor type B agonist baclofen was shown to inhibit TLOSRs, thereby significantly decreasing acid reflux after a meal in healthy controls. ${ }^{11}$ Inhibitory $\mathrm{GABA}_{\mathrm{B}}$ receptors are present at many levels of the vago-vagal pathway that organises TLOSRS.

In this issue of Gut, Zhang and colleagues $^{12}$ demonstrated that acute administration of baclofen reduced the rate of TLOSRs and reflux events by approximately $40 \%$ in a group of patients with reflux oesophagitis (see page 19). Based on these findings, the authors concluded that reduction in the rate of TLOSRs is an appropriate target in GORD. In addition, they suggest that $\mathrm{GABA}_{\mathrm{B}}$ agonists may be useful as therapeutic agents for the management of reflux disease. As baclofen is already in use for the long term treatment of spasticity, in higher doses than used in the present study, there is a potential for clinical application in GORD in the not so distant future. However, in spite of the reduction in the number of reflux episodes, baclofen caused no significant decrease in postprandial oesophageal acid exposure in the present study. The lack of a significant effect on acid exposure is at least partly related to patient selection, as many patients had only minimal amounts of acid reflux during the placebo arm of the study. Confirmation of a clinically relevant effect of baclofen in GORD therefore awaits long term studies, with efficacy assessment on both reflux related symptoms and prolonged $\mathrm{pH}$ monitoring, in patients with a high rate of TLOSR related acid reflux events.

Although initial postprandial motility studies, performed in the right supine position, reported an increased rate of TLOSRs in patients with GORD, ${ }^{3}{ }^{4}$ recent postprandial studies in the more physiological upright position show a similar rate of TLOSRs in healthy controls and patients with GORD. ${ }^{5-7}$ A more consistent finding among different studies is the higher prevalence of acid reflux during TLOSRs in patients with reflux disease. Because TLOSRs are the major mechanism of reflux, inhibition of TLOSRs has the potential to provide a substantial reduction in acid reflux events in GORD.

Acid suppressive therapy is most successful in patients with mild to moderate reflux disease with predominantly upright reflux, and less successful in patients with more severe reflux oesophagitis and patients with supine reflux. ${ }^{13-16}$ As TLOSR related acid reflux is predominant in patients with mild to moderate GORD, treatment of these patients by inhibiting TLOSRs may provide an alternative to acid suppression. Furthermore, baclofen or related agents could be used as add-on therapy in GORD patients with incomplete relief by acid suppression and/or in patients with more severe GORD. In about half of patients with persisting reflux symptoms, in spite of standard proton pump inhibitor therapy, refractory acid reflux can be demonstrated which often responds to a further increase in proton pump inhibitor dose. ${ }^{15} 16$ In the remaining patients, symptoms may be related to ongoing non-acid reflux, for which treatment options are limited. ${ }^{17}$ As recent data suggest that TLOSRs are the principal mechanism underlying non-acidic reflux episodes as well, ${ }^{7}$ assessment of the therapeutic potential of baclofen or related compounds under these specific circumstances seems warranted.

The relevance of TLOSRs in the pathophysiology of GORD may be more related to what is present at the level of the cardia, at the moment of a TLOSR, than to their frequency. Postprandial gastric acid secretion and the composition and/or distribution of the fundic contents appear to be critical variables in the development of GORD. Therefore, the most pathophysiologically oriented strategy should aim to minimise the number of "harmful" TLOSRs associated with acid reflux by both reducing the triggering of TLOSRs and modifying the composition and/or distribution of gastric fundic contents.

The paper by Zhang et al demonstrates that inhibition of TLOSRs with baclofen reduces the frequency of acid reflux in GORD patients. Both acid suppressive 
therapy and reduction of TLOSRs address one important factor of the pathophysiology of GORD. Because the major functional abnormality in reflux disease appears to be the occurrence of acid reflux during TLOSRs, both strategies are not exclusive and may become complementary in the near future.

Gut 2002;50:6-7

\section{Authors' affiliations}

J Tack, D Sifrim, Centre for

Gastroenterological Research, University of

Leuven, Leuven, Belgium

Correspondence to: Dr J Tack, Department of Internal Medicine, Division of Gastroenterology, University Hospital Gasthuisberg, Herestraat 49 B-3000 Leuven, Belgium;

Jan.Tack@med.kuleuven.ac.be

\section{REFERENCES}

1 Ghillebert G, Demeyere AM, Janssens J, et al. How well can quantitative 24-hour intraesophageal $\mathrm{pH}$ monitoring distinguish various degrees of reflux disease? Dig Dis $\mathrm{Sci}$ 1995:40:1317-24.

2 Hirschowitz BI. A critical analysis, with appropriate controls, of gastric acid and pepsin secretion in clinical esophagitis. Gastroenterology 1991;101:1149-58.
3 Dodds WJ, Dent J, Hogan WJ, et al. Mechanisms of gastroesophageal reflux in patients with reflux esophagitis. N Engl J Med 1982;307: 1547-52.

4 Holloway RH, Kocyan P, Dent J. Provocation of transient lower esophageal sphincter relaxations by meals in patients with symptomatic gastroesophageal reflux. Dig Dis Sci 1991;36:1034-9.

5 Schoeman MN, Tippett MD, Akkermans LMA, et al. Mechanisms of gastroesophageal reflux in ambulant healthy human subjects. Gastroenterology 1995;108:83-91.

6 Penagini R, Schoeman MN, Dent J, et al. Motor events underlying gastro-esophageal reflux in ambulant patients with reflux esophagitis. Neurogastroenterol Motil 1996:8:131-41.

7 Sifrim D, Holloway R, Silny J, et al. Composition of the postprandial refluxate in patients with gastroesophageal reflux disease. Am J Gastroenterol 2001;96:647-55.

8 Van Herwaarden MA, Samsom M, Smout AJ. Excess gastroesophageal reflux in patients with hiatus hernia is caused by mechanisms other than transient LES relaxations. Gastroenterology 2000;1 19:1439-46.

9 Mittal RK, Balaban DH. The esophagogastric junction. N Engl J Med 1997;336:924-32.

10 Holloway RH, Dent J. Medical treatment of gastroesophageal reflux disease-beyond the proton pump inhibitors. Dig Dis 2000; 18:7-13.

11 Lidums I, Lehmann A, Checklin $\mathrm{H}$, et al. Control of transient lower esophageal sphincter relaxations and reflux by the GABA(B) agonist baclofen in normal subjects. Gastroenterology 2000;1 18:7-13.
12 Zhang Q, Lehmann A, Rigda R, et al. Control of transient lower oesophageal sphincter relaxations and reflux by the $G A B A_{B}$ agonist baclofen in patients with gastro-oesophageal reflux disease. Gut 2001;50:19-24.

13 Dehn TCB, Sheperd HA, Colin-Jones D, et al. Double blind comparison of omeprazole versus cimetidine in the treatment of symptomatic erosive reflux oesophagitis, assessed endoscopically, histologically and by $24 \mathrm{~h} \mathrm{pH}$ monitoring. Gut 1990;31:509-13.

14 Hetzel DJ, Dent J, Reed WD, et al. Healing and relapse of severe peptic esophagitis after treatment with omeprazole. Gastroetnerology 1988;95:903-12.

15 Klinkenberg-Knol EC, Meuwissen SGM. Combined gastric and esophageal 24-hour $\mathrm{pH}$ monitoring and esophageal manometry in patients with reflux disease resistant to treatment with omeprazole. Aliment Pharmacol Ther 1990:4:485-95.

16 Holloway RH, Dent J, Narielvala F, et al. Relation between oesophageal acid exposure and healing of oesophagitis with omeprazole. Gut 1996;38:649-54

17 Vela M, Camacho-Lobato L, Hatlebakk J, et al. Effect of omeprazole (PPI) on ratio of acid to nonacid gastroesophhageal reflux. Studies using simultaneous intraesophageal impedance and $\mathrm{pH}$ measurement. Gastroenterology 1999:116:A209.

18 Demedts I, Tack J, Koek G, et al. Gastro-esophageal reflux disease refractory to proton pump inhibitors: acid, bile reflux, or both? Gastroenterology 1999;116:A326.

\section{Positive suggestions about the anti-HBc positive donor}

\section{Mutimer}

\section{What to do with the anti-hepatitis B virus core antigen positive donor liver}

T he paper by Roque-Afonso et al in this issue of Gut $^{1}$ describes the outcome of recipients following transplantation of livers from organ donors who have detectable antibody to the hepatitis $\mathrm{B}$ virus (HBV) core antigen $(\mathrm{HBc})$ but undetectable serum hepatitis B surface antigen (anti-HBc positive, HBsAg negative) (see page 95). Serum anti-HBc positivity simply confirms exposure to $\mathrm{HBV}$. Its presence in the absence of serum HBsAg identifies the patient with resolved infection. Frequently, antibodies to HBsAg (anti-HBs) will be absent, fading in titre for many patients from the time of acute infection. Thus the patient with a past resolved $\mathrm{HBV}$ infection will be anti-HBc positive and HBsAg negative, and anti-HBs may or may not be present. Irrespective of the anti-HBs status of the donor, it is known that the use of anti-HBc positive (HBsAg negative) donor livers will transmit HBV to the recipient and will establish chronic infection and graft damage. ${ }^{2-5}$ This is termed de novo HBV infection. Occasionally, aggressive infection with graft failure and patient death may be observed. ${ }^{6}$ In general, the other organs of the anti-HBc positive donor can be used with little risk of de novo infection to the recipient. ${ }^{2}$ This observation suggests that potentially infectious $\mathrm{HBV}$ is present in the liver, but not in other organs, and that reactivation of the virus occurs following transplantation in the context of immunosuppression.

There is considerable geographic variation in the frequency of organ donor anti-HBc positivity. In the UK, reflecting a relatively low lifetime risk of exposure to $\mathrm{HBV}$, donor anti-HBc positivity is uncommon. However, de novo infection causes significant morbidity for the few unlucky recipients. ${ }^{4}$ In Southern Europe and Japan, the frequency is high, reflecting past exposure to $\mathrm{HBV}$ of a large proportion of the potential organ donor pool. ${ }^{57}$ Of consecutive organ donors used by the liver transplant surgeons at Hospital Paul Brousse, 22/315 (7\%) were anti-HBc positive. RoqueAfonso et al from Hospital Paul Brousse present the pretransplant HBV serological status and post-transplant outcome (with or without passive immunoprophylaxis) of the 22 recipients of those anti-HBc positive liver donations. The results are nicely organised and presented in table 1 of their manuscript. Detailed analysis of the data in the table emphasises the important and accepted principles that should be applied to the use of anti-HBc positive livers.

Patient Nos 1-4 are the ideal recipients for these organs. These recipients had HBV associated liver failure as the indication for liver transplantation and required immunoprophylaxis to prevent graft reinfection by HBV. Under this circumstance, it appears that immunoprophylaxis prevented both reinfection and de novo infection by HBV. Thus with no added cost or effort, the anti-HBc positive liver was suitably and safely placed.

Patient Nos 19-22 provide confirmation that the anti-HBc positive liver, when placed into a recipient who lacks pretransplant HBV immunity and who 
does not receive post-transplant prophylaxis, will establish de novo infection in the majority of instances.

The outcome of patient Nos 5-9 suggests that passive immunoprophylaxis can prevent de novo infection of most non-immune recipients (only $1 / 5$ treatment failures observed). A similar beneficial impact was observed by Uemoto et al who used immunoprophylaxis for three paediatric recipients of living related anti-HBc positive livers. ${ }^{5}$ Recent published work suggests that lamivudine prophylaxis, with or without immunoprophylaxis, may also prevent de novo recipient infection in this at-risk group. ${ }^{8} 9$ Yu et al have published a small study using lamivudine (without immunoprophylaxis) to prevent de novo infection of nine recipients of anti-HBc positive livers. However, four recipients were HBV immune pretransplant and three of the remaining patients died soon after transplantation. Thus a sustained effect of lamivudine prophylaxis for nonimmune recipients of anti-HBc positive livers was confirmed for only three patients. ${ }^{9}$ Dodson et al reported successful results with the combination of lamivudine and immunoglobulin given to 15 recipients, of whom seven were non-immune. ${ }^{8}$ Compared with immunoprophylaxis, lamivudine is inexpensive and easier to administer. Immunoglobulin is plasma derived and therefore its use raises microbiological concerns. Thus patient and doctor acceptance of lamivudine is superior, and compliance with lamivudine prophylaxis is good.

The outcome of patient Nos 10-18 poses more questions than provides answers. These recipients were either anti-HBc or anti-HBs positive pretransplant, most received immunoprophylaxis, and none developed de novo infection. However, the contribution of passive immunoprophylaxis to the favourable outcome of these recipients cannot be determined. Patient Nos 10-16 may have been protected from de novo infection by their own pre-existing HBV immunity. For these recipients, immunoprophylaxis may represent an unnecessary and costly addition to their care. Indeed, the outcome of patient Nos 17 and 18, who were anti-HBs positive pretransplant, who did not receive prophylaxis, and who did not experience de novo infection, suggests that anti$\mathrm{HBC}$ positive livers may be suitably placed (without the need for prophylaxis) in recipients who have serological evidence of HBV immunity. Other reports support this possibility that recipient anti-HBs and/or anti-HBc positivity may prevent de novo infection from anti-HBc positive livers. ${ }^{7}$

In summary, the data presented by Roque-Afonso et al in this issue of Gut support the following conclusions and recommendations.

- The use of anti-HBc positive livers without prophylaxis for non-immune recipients can and must be avoided (it follows that the anti-HBc status of the donor and recipient must be known).

-If possible, the anti-HBc positive liver should be used for a HBsAg positive recipient (who receives the combination of lamivudine and immunoprophylaxis to prevent graft reinfection and de novo infection). If a liver unit does not have a suitable HBsAg positive recipient for the anti-HBc positive donor, then the donor liver might be offered to a unit that does.

- For the HBsAg negative recipient of the anti-HBc positive liver, it appears that pretransplant immunity to HBV, immunoprophylaxis, or lamivudine (or combinations) significantly reduce the risk of de novo infection. Probably, and despite the fact that conclusive efficacy data are scarce, lamivudine without immunoprophylaxis will be preferred by many transplant units.
Above all, the anti-HBc positive liver can and must be used. In these times of donor organ shortage, a suitable and grateful recipient can always be found.

Gut 2002;50:7-8

\section{Author's affiliation}

D Mutimer, Liver and Hepatobiliary Unit, Queen Elizabeth Hospital, Birmingham, UK

Correspondence to: D Mutimer;

david.mutimer@university_b.wmids.nhs.uk

\section{REFERENCES}

1 Roque-Afonso AM, Feray C, Samuel D, ef al. Antibodies to hepatitis $B$ surface antigen prevent viral reactivation in recipients of liver grafts from anti-HBc positive donors. Gut 2001;50:95-9.

2 Wachs ME, Amend WJ, Ascher NL, et al. The risk of transmission of hepatitis $B$ from $\mathrm{HBsAg}$ $(-), \mathrm{HBcAb}(+), \mathrm{HB} \lg M(-)$ organ donors. Transplantation 1995;59:230-4.

3 Dickson RC, Everhart JE, Lake JR, et al. Transmission of hepatitis $B$ by transplantation of livers from donors positive for antibody to hepatitis B core antigen. The National Institute of Diabetes and Digestive and Kidney Diseases Liver Transplantation Database. Gastroenterology 1997;113:1668-74.

4 Gow PJ, Mutimer DJ. De novo hepatitis B infection acquired during liver transplantation. Q J Med 2001;94:271-5.

5 Uemoto S, Sugiyama K, Marusawa H, et al. Transmission of hepatitis $B$ virus from hepatitis $B$ core antibody-positive donors in living related liver transplants. Transplantation 1998:65:494-9.

6 Segovia R, Sanchez-Fueyo A, Rimola A, et al. Evidence of serious graft damage induced by de novo hepatitis $B$ virus infection after liver transplantation. Liver Transpl

2001;7:106-12.

7 Prieto $M$, Gomez MD, Berenguer M, et al. De novo hepatitis $B$ after liver transplantation from hepatitis $B$ core antibody-positive donors in an area with high prevalence of anti-HBc positivity in the donor population. Liver Transpl 2001:7:51-8.

8 Dodson SF, Bonham CA, Geller DA, et al. Prevention of de novo hepatitis $B$ infection in recipients of hepatic allografts from anti-HBc positive donors. Transplantation 1999:68:1058-61.

9 Yu AS, Vierling JM, Colquhoun SD, et al Transmission of hepatitis $B$ infection from hepatitis B core antibody-positive liver allografts is prevented by lamivudine therapy. Liver Transpl 2001;7:513-7. 